\title{
Deficits in hippocampal-dependent memory across different rodent models of early life stress: systematic review and meta-analysis
}

\author{
Mariana Rocha', Daniel Wang ${ }^{1}$, Victor Avila-Quintero ${ }^{2}$, Michael H. Bloch ${ }^{1,2}$ and Arie Kaffman (i)
}

\begin{abstract}
Exposure to early life stress (ELS) causes abnormal hippocampal development and functional deficits in rodents and humans, but no meta-analysis has been used yet to quantify the effects of different rodent models of ELS on hippocampal-dependent memory. We searched PubMed and Web of Science for publications that assessed the effects of handling, maternal separation (MS), and limited bedding and nesting (LBN) on performance in the Morris water maze (MWM), novel object recognition (NOR), and contextual fear conditioning (CFC). Forty-five studies met inclusion criteria ( $n=451-763$ rodents per test) and were used to calculate standardized mean differences (Hedge's $g$ ) and to assess heterogeneity, publication bias, and the moderating effects of sex and species (rats vs. mice). We found significantly lower heterogeneity in LBN compared to handling and MS with no consistent effects of sex or species across the three paradigms. LBN and MS caused similar cognitive deficits in tasks that rely heavily on the dorsal hippocampus, such as MWM and NOR, and were significantly different compared to the improved performance seen in rodents exposed to handling. In the CFC task, which relies more on the ventral hippocampus, all three paradigms showed reduced freezing with moderate effect sizes that were not statistically different. These findings demonstrate the utility of using meta-analysis to quantify outcomes in a large number of inconsistent preclinical studies and highlight the need to further investigate the possibility that handling causes different alterations in the dorsal hippocampus but similar outcomes in the ventral hippocampus when compared to MS and LBN.
\end{abstract}

\section{Introduction}

Early life stress (ELS) impairs the development of several interconnected brain regions including the hippocampus, prefrontal cortex, striatum, and amygdala ${ }^{1,2}$. Of these, reduced hippocampal volume and abnormal hippocampal function are some of the most reproducible findings in individuals exposed to $\operatorname{ELS}^{1,3,4}$. This is not surprising given that the hippocampus undergoes substantial developmental changes during childhood that are highly sensitive to stress ${ }^{5-7}$. The hippocampus also forms extensive connections with other brain regions involved

\footnotetext{
Correspondence: Arie Kaffman (arie.kaffman@yale.edu)

${ }^{1}$ Department of Psychiatry, Yale University School of Medicine, 300 George Street, Suite 901, New Haven, CT 06511, USA

${ }^{2}$ Child Study Center, Yale University School of Medicine, 230 South Frontage Road, New Haven, CT 06519, USA
}

in declarative memory, spatial learning, threat detection, stress reactivity, and mood regulation ${ }^{8,9}$. These features make the hippocampus a potentially important hub in mediating several of the cognitive, emotional, and physiological abnormalities seen in individuals exposed to ELS $^{1,3,4}$.

Elucidating the mechanisms by which ELS affects hippocampal development is a challenging task in humans because of the inherent complexity and heterogeneity of the adversities, genetic variability, and numerous additional variables that are difficult to control in clinical settings ${ }^{10}$. In addition, causally linking structural and functional changes in the hippocampus with alterations in anxiety, stress reactivity, or cognition are practically impossible to do in humans. Nevertheless, the conserved nature by which the hippocampus develops in 
mammalian species ${ }^{5,11}$ and the observation that rodent models of ELS cause significant impairment in its development and long-term function ${ }^{10}$ suggest that work in rodents may clarify important details about the role that abnormal hippocampus development plays in the longterm consequences of ELS. Indeed, an elegant body of work has shown that chronically elevated levels of the neuropeptide corticotrophin-releasing hormone (CRH) mediate the synaptic and hippocampal-dependent memory deficits in rodents exposed to a rodent model of ELS known as limited bedding and nesting $(\mathrm{LBN})^{12}$.

Several key questions regarding the effects of ELS on hippocampal function in rodents remain unresolved. For example, it is currently unclear whether some types of ELS lead to more severe hippocampal-dependent deficits, whether males and females are equally sensitive, and if some tests are more sensitive for detecting hippocampaldependent cognitive deficits caused by ELS. Even within the same ELS paradigm, different research groups report variable outcomes and the literature is replete with examples of inconsistent findings ${ }^{10,13,14}$. These inconsistencies appear to be related to many factors such as different genetic backgrounds, poor standardization of procedures across labs, and other stochastic variables ${ }^{10,14-16}$. To address these issues, we conducted a systematic review and subsequent meta-analysis examining the effects of three types of ELS: handling (also known as brief maternal separation), maternal separation (MS), and LBN on hippocampal function in rodents (mice and rats). Hippocampal-dependent memory was assessed using 3 behavioral tests: (1) the Morris water maze (MWM), (2) Novel Object Recognition (NOR), and (3) Contextual Fear Conditioning (CFC). We chose these tests because they all require normal hippocampal function ${ }^{8,17,18}$ and are commonly tested in rodents exposed to ELS ${ }^{13,14}$. Moreover, the MWM and the NOR rely more on the dorsal hippocampus ${ }^{8,19-21}$, whereas freezing behavior in the CFC is thought to have a strong ventral hippocampus component ${ }^{8,22-25}$. Nevertheless, performance in these tests, especially steps that involve consolidation and retrieval, also require the prefrontal cortex and other cortical regions that need to be considered when interpreting behavioral outcomes in these tests ${ }^{17,18,26-28}$.

Although two systematic reviews have previously examined the effects of different types of ELS on behavioral outcomes in rodents, including spatial learning ${ }^{13,14}$ they were qualitative in nature and did not include a meta-analysis. Meta-analysis is routinely used in clinical settings but has rarely been used to address conflicting results in preclinical studies in rodents ${ }^{29}$. In fact, we are aware of only two meta-analyses examining the effects of ELS on behavioral outcome in rodents, one assessing pain sensitivity $^{30}$ and the other examining anxiety-likebehavior $^{31}$. No meta-analysis to date has assessed the effects of ELS on spatial learning and none of the available meta-analysis included the LBN which is one of the most commonly used ELS paradigms in rodents ${ }^{32}$.

The primary goals of this meta-analysis were to compare the effect sizes and heterogeneity of the three different ELS paradigms in the MWM, NOR, and CFC. We also examined the effects of several moderators such as sex, species (i.e., mice vs. rats), separation index (i.e., the number of days pups were separation $\mathrm{X}$ length of the separation), and temperature of isolation on cognitive outcomes. Lastly, we assessed for possible publication bias and its impact on behavioral outcomes in these tests.

\section{Methods}

\section{Search strategy}

Two reviewers (MR, DW) searched the electronic databases of PubMed and Web of Science on September 30th, 2019 for relevant studies using the following searches: (early life stress OR ELS OR postnatal stress OR maternal separation OR neonatal stress OR limited bedding and nesting OR LBN OR brief maternal separation OR BMS OR handling) AND (mice OR mouse OR mus musculus OR rats OR rat) AND (Morris water maze OR MWM OR novel object recognition OR NOR OR novel object location OR NOL OR contextual fear conditioning OR CFC). Studies were limited to English language articles. Additional citations came from previous systematic reviews ${ }^{13,14,32,33}$. Studies obtained from the search, the titles, and abstracts were examined by the two reviewers (MR, DW) to determine preliminary inclusion. Discrepancies were addressed by the reviewers through discussion and through conversation with senior reviewers (AK, MHB). Despite a concerted effort to identify all relevant studies, it is possible that we have missed some studies due to the enormity of the literature and the different names used to describe these paradigms. In addition, we did not register this study with PROSPERO prior to extracting the data and therefore are not able to do this at this point. However, we are confident that no previous meta-analyses have examined this question before.

\section{Study selection and data extraction}

After preliminary inclusion, studies were carefully read to determine if inclusion criteria were met. See Supplemental Information for additional details regarding selection criteria and data extraction are available in the supplementary information.

\section{Data analysis}

Statistical Analyses were conducted using the Comprehensive Meta-Analysis Version 3.0 software (Biostat, 2016). Outcomes of interest were measures of hippocampal-dependent Memory in the MWM, NOR, and CFC. Details regarding specific behavioral measurements 
are available in the supplementary information. Hedge's g was used as the pooled measurement of effect size as it is preferred over Cohen's d for small samples, which are common in animal studies ${ }^{34}$. Both fixed and random effects were calculated and are presented in the results section. Random effect analysis was used as the primary analysis to account for the large heterogeneity between studies. Fixed-effect analysis was conducted as a sensitivity analysis because random-effects models may be overly conservative in situations where a relatively small number of studies is available. Publication bias was assessed using funnel plots and the Egger's test, and heterogeneity was measured utilizing the $I^{2}$ statistics and Chi-square test for heterogeneity ${ }^{35,36}$. Details regarding the analyses for moderating effects of sex, species, separation index and temperature are available in the supplemental information.

\section{Results}

\section{Selection studies}

Figure S1 depicts the selection strategy for included studies. In total, 1435 articles were identified for consideration, of which 45 studies were eligible for inclusion. Reasons for exclusion of studies are identified in the Supplemental Information Fig. S1. Table 1 describes the characteristics of our included studies and additional details are available in the supplemental information Tables S1 and S2.

\section{Morris water maze (MWM): latency to find platform training}

A forest plot summary of the effects of handling (7 studies, $n=162$ rodents), MS (15 studies, $n=338$ rodents), and LBN (11 studies, $n=243$ rodents) on spatial learning in the MWM is shown in Fig. 1 and Fig. S2. Test for subgroup differences demonstrated significant differences in effect size (Hedge's $g$ ) between the three experimental paradigms (random effect: $X^{2}=7.34, \mathrm{df}=2$, $p=0.03$; fixed effect: $\chi^{2}=29.3, \mathrm{df}=2, p<0.0005$ ). Posthoc pairwise comparisons found significant differences in effect sizes between handling and MS (random effect: $\chi^{2}=5.81 \mathrm{df}=1, p=0.02$; fixed effect: $\chi^{2}=22.9 \mathrm{df}=1$, $p=<0.0005$ ) and between handling and LBN (random effect: $\chi^{2}=7.3, \mathrm{df}=1, p=0.01$; fixed effect: $\chi^{2}=26.3$, $\mathrm{df}=1, p<0.0005$ ) but not between MS and LBN (random effect: $\chi^{2}=0.06 \mathrm{df}=1, p=0.81$; fixed effect: $\chi^{2}=0.44$ $\mathrm{df}=1, p=0.51$ ).

Exposure to handling reduced the latency to find the platform (Fig. 1, top and Fig. S2), an outcome that was not significant for random effect (Hedge's $g=0.58 \pm 0.36$, 95\% Confidence Interval $(\mathrm{CI})=-0.12-(+1.28), z=1.62, p=$ $0.11, k=7)$ but significant using fixed-effect analysis (Hedge's $g=0.58 \pm 0.16,95 \%$ Confidence Interval $(\mathrm{CI})=$ $0.27-0.89, z=3.70, p=0.0002, k=7)$. There was significant heterogeneity between studies $\left(I^{2}=80 \%, Q=30\right.$, $\mathrm{df}=6, p<0.0005)$ but no evidence for publication bias based on funnel plot asymmetry or Egger's test for handling (Supplemental information Fig. S3A), Egger's test $p=0.97)$. Sex $(Q=1.45, \mathrm{df}=1, p=0.22)$ or species (i.e., rats vs. mice, $Q=0.00, \mathrm{df}=1, p=0.99$ ) did not affect latency to find the platform.

In contrast to the reduced latency seen in handling, exposure to MS was associated with a significant increase in the latency to find the platform (random effect: Hedge's $g=-0.39 \pm 0.18, \quad 95 \% \mathrm{CI}=-0.74-(-0.03), \quad z=-2.14$, $p=0.032, k=15$; fixed effect: Hedge's $g=-0.30 \pm 0.11$, $95 \% \mathrm{CI}=-0.52-(-0.09), \quad z=-2.75, p=0.006, k=15$, Fig. 1, middle). There was significant heterogeneity between studies $\left(I^{2}=62 \%, \mathrm{Q}=37, \mathrm{df}=14, p=0.0007\right)$ with no evidence of publication bias based on the funnel plot (Supplemental information Fig. S3B) or the Egger's test $(p=0.14)$. Stratified subgroup analysis demonstrated no significant effect of $\operatorname{sex}(Q=1.9, \mathrm{df}=1, p=0.17)$, and all MS studies were done in rats, preventing us from assessing the effect of species on latency to find the platform. Moderator analyses found no significant effect of separation index $(\beta=-0.0063 \pm 0.0063,95 \% \mathrm{CI}=$ $-0.019-(0.062), Z=-0.99, p=0.32, k=15)$, but significant effect of separation temp $(\beta=-0.44 \pm 0.18,95 \%$ $\mathrm{CI}=-0.8-(-0.079), Z=-2.39, p=0.017, k=15)$ with longer latency to find the platform associated with incubation at a higher temperature during the MS procedure.

Similar to outcomes seen in MS, rodents exposed to LBN showed a significant increase in latency to find the platform (random and fixed effects: Hedge's $g=-0.44 \pm$ $0.12,95 \% \mathrm{CI}=-0.68-(-0.20), z=-3.59, p<0.0005, \mathrm{k}=$ 11; Fig. 1, bottom). The heterogeneity between LBN studies was minimal and non-significant $\left(I^{2}=0 \%, Q=6.9\right.$, $\mathrm{df}=10, p=0.73$ ). There was no evidence for publication bias based on funnel plot symmetry and a non-significant Egger's test $(p=0.35$, Supplemental information Fig. S3C). No significant effect of sex was seen in LBN studies $(Q=1.32, \mathrm{df}=1, p=0.25)$, but there was a significant effect of species $(Q=4.66, \mathrm{df}=1, p=0.033)$. Further examination revealed that LBN increased latency to find the platform in rats (Hedge's $g=-0.73 \pm 0.18,95 \% \mathrm{CI}=$ $-1.1-(-0.4), z=-4.02, p<0.0005, k=5)$, but not in mice (Hedge's $g=-0.20 \pm 0.17,95 \% \mathrm{CI}=-0.53-(+0.12)$, $z=-1.2, p=0.23, k=6)$.

\section{MWM: probe trial}

The forest plot summary for the effects of handling (2 studies, $n=60$ rodents), MS (15 studies, $n=343$ rodents), and LBN (7 studies, $n=162$ rodents) on performance in the probe trial of the MWM are shown in Fig. 2. Rodents exposed to MS and LBN spent significantly less time swimming in the correct quadrant whereas handling was associated with a non-significantly increased time spent in the correct quadrant compared to controls. Tests 
Table 1 Alphabetical list of studies included in the meta-analysis.

\begin{tabular}{|c|c|c|c|c|c|}
\hline Reference & ELS paradigm & Species-strain & Sex & Sample size & Outcome(s) tested \\
\hline Aisa, $2007^{45}$ & MS & Rat-Wistar & M & 10-12/group & MWM, NOR \\
\hline Asia, $2008^{46}$ & MS & Rat-Wistar & $\mathrm{F}$ & 10/group & NOR \\
\hline Banqueri, $2018^{47}$ & MS & Rat-Wistar & $\mathrm{F}$ & 10/group & MWM \\
\hline Baudin, $2012^{48}$ & MS & Rat-LH & M & 12/group & MWM \\
\hline Burnson, $2005^{49}$ & LBN & Rat-SD & M & 8-11/group & MWM, NOR \\
\hline Cao, $2014^{50}$ & MS & Rat-SD & M & 9-10/group & MWM \\
\hline Chocyk, 2014 & MS & Rat-Wistar & $\mathrm{M}$ and $\mathrm{F}$ & 20/group & CFC \\
\hline Couto-Pereira, $2019^{52}$ & MS, Handling & Rat-Wistar & M & 13/group & CFC \\
\hline Cui, $2006^{53}$ & LBN & Rat-SD & M & 8/group & MWM \\
\hline Dalle, $2017^{54}$ & MS & Rat-SD & M & 10-20/group & MWM \\
\hline Diehl, $2012^{55}$ & MS & Rat-Wistar & M & 9/group & MWM \\
\hline Diehl, $2014^{56}$ & MS & Rat-Wistar & $M$ and $F$ & 8/group & CFC \\
\hline Fegnolio, $2005^{40}$ & Handling & Rat-SD & M & 8-11/group & MWM, NOR \\
\hline Guijarro, $2007^{57}$ & MS, Handling & Rat-Wistar & M & 14-24/group & CFC \\
\hline Hoeijmakers, $2018^{58}$ & LBN & Mouse-C57B1/6」 & M & 9-12/group & MWM, NOR \\
\hline Huang, $2002^{59}$ & MS & Rat-SD & M & 8-9/group & MWM \\
\hline Ivy, $2010^{60}$ & LBN & Rat-SD & M & 13-23/group & MWM, NOR \\
\hline Kanatsou, $2017^{61}$ & LBN & Mouse, C57Bl6, C57BL/6N & M & 10/group & CFC \\
\hline Kosten, $2006^{62}$ & MS, Handling & Rat-SD & $\mathrm{M}$ and $\mathrm{F}$ & 6-8/group & CFC \\
\hline Lai, $2006^{63}$ & MS & Rat-SD & M & $20-22$ & MWM \\
\hline $\mathrm{Li}, 2018^{64}$ & Handling & Mouse-BALB/cCrSlc & M & 8-10/group & MWM \\
\hline Manzano-Nieves, $2018^{65}$ & LBN & Mouse-C57BL/6N & $M$ and $F$ & 7-14/group & CFC \\
\hline Molet, $2016^{66}$ & LBN & Rat-SD & M & 6/group & NOR \\
\hline Naninik, $2015^{67}$ & LBN & Mouse-C57B1/6J & $\mathrm{M}$ and $\mathrm{F}$ & 6/group & MWM, NOR \\
\hline Naninik, $2017^{68}$ & LBN & Mouse-C5BI/6J & M & 13-14/group & MWM, NOR \\
\hline Noschang, $2010^{69}$ & Handling & Rat-Wistar & $\mathrm{M}$ and $\mathrm{F}$ & 7-10/group & MWM \\
\hline Plescia, $2014^{70}$ & Handling & Rat-Wistar & $\mathrm{F}$ & 16-26/group & MWM, NOR \\
\hline Pusceddu, $2015^{71}$ & MS & Rat-SD & $\mathrm{F}$ & 10/group & NOR \\
\hline Reshetnikov, $2018^{72}$ & MS, Handling & Mouse-c57B1/6 & M & 8-10/group & NOR \\
\hline Rice, $2008^{73}$ & LBN & Mouse-C57BL/6J & M & 6-13/group & MWM, NOR \\
\hline Solas, $2010^{74}$ & MS & Rat-Wistar & M & 15/group & MWM, NOR \\
\hline Sun, $2014^{75}$ & MS & Rat-Wistar & $M$ and $F$ & 7-13/group & MWM, CFC \\
\hline Uysal, $2005^{76}$ & MS & Rat-Wistar & $\mathrm{M}$ and $\mathrm{F}$ & 8/group & MWM \\
\hline Wang L., $2011^{77}$ & MS & Mouse-BALB/CJ & $\mathrm{F}$ & 16/group & NOR \\
\hline Wang XD Rammes, $2011^{78}$ & LBN & Mouse, $129 S 2 / S v$ X C57BL/6J & M & 18-20/group & MWM \\
\hline Xiong, $2014^{79}$ & MS & Rat-SD & $\mathrm{F}$ & 8/group & CFC \\
\hline Xiong, $2015^{80}$ & MS & Rat-SD & M & 12-16/group & MWM, CFC \\
\hline$X u, 2018^{81}$ & MS & Rat-SD & $\mathrm{M}$ and $\mathrm{F}$ & 8/group & MWM \\
\hline Xue, $2013^{82}$ & MS & Rat-Wistar & M & 6/group & MWM \\
\hline
\end{tabular}


Table 1 continued

\begin{tabular}{|c|c|c|c|c|c|}
\hline Reference & ELS paradigm & Species-strain & Sex & Sample size & Outcome(s) tested \\
\hline Zaharia, $1996^{83}$ & Handling & Mouse, BALB/cByJ, C57BL/6ByJ & M & 16-18/group & MWM \\
\hline Zhang, $2014^{84}$ & MS & Rat-SD & M & 11-13/group & MWM \\
\hline Zoicas, $2016^{85}$ & MS & Mouse-CD1 & M & 12/group & NOR \\
\hline
\end{tabular}

LH Long-Havens, M Males, F Females, SD Sprague-Dawley.

For additional details see Tables S1 and S2 in the Supplemental Information.

for subgroup differences demonstrated significant differences in overall effect sizes between the three experimental paradigms (random effect: $\chi^{2}=6.99, \mathrm{df}=2, p=$ 0.03; fixed effect: $\chi^{2}=14.9, \mathrm{df}=2, p=0.001$ ) that was due to increased time swimming in the correct quadrant in handling compare to MS (random effect: $\chi^{2}=5.45, \mathrm{df}=1$, $p=0.02$; fixed effect: $\left.\chi^{2}=11.9, \mathrm{df}=1, p=0.0006\right)$ and LBN (random effect: $\chi^{2}=6.63 \mathrm{df}=1, p=0.01$; fixed effect: $\left.\chi^{2}=14.3, \mathrm{df}=1, p<0.0005\right)$. Comparison between MS and LBN was not significant using either the randomeffects $\left(\chi^{2}=0.57, \mathrm{df}=1, p=0.45\right)$ or fixed-effects models $\left(\chi^{2}=1.02 \mathrm{df}=1, p=0.31\right)$.

Since only two handling studies were included (Table S2), the outcomes for the probe trial should be considered exploratory and did not include analyses of publication bias or on the effects of sex and species. Nevertheless, handling increased time swimming in the correct quadrant (Fig. 2, top), an outcome that was not significant using random effect (Hedge's $g=0.72 \pm 0.49,95 \% \mathrm{CI}=$ $-0.25-(+1.69), z=1.5, p=0.14, k=2)$ but significant using a fixed-effect analysis (Hedge's $g=0.58 \pm 0.27,95 \%$ $\mathrm{CI}=0.06-1.10, z=2.2, p=0.029, k=2)$. The heterogeneity between the studies was large but did not reach statistical significance given the small number of studies $\left(I^{2}=65 \%, Q=3, \mathrm{df}=1, p=0.091\right)$.

Exposure to MS reduced time swimming in the correct quadrant (random effect: Hedge's $g=-0.53 \pm 0.21,95 \%$ CI $=-0.93-(-0.12), z=-2.6, p=0.01, k=15$; fixed effect: Hedge's $g=-0.41 \pm 0.11,95 \% \mathrm{CI}=-0.62-(-0.20), \quad z=$ $-3.8, p=0.00014, k=15$, Fig. 3 middle). The heterogeneity between the studies was large and statistically significant $\left(I^{2}=72 \%, Q=50, \mathrm{df}=14, p<0.0005\right)$. Funnel plot asymmetry suggested a possible publication bias (Supplemental information Fig. S4A), a finding that was confirmed using the Egger's test $(p=0.009)$. Nevertheless, the effect of MS remained significant after adjusting for funnel plot asymmetry using Duval and Tweedie's Trim-and-Fill method, Hedge's $g=-0.30,95 \% \mathrm{CI}=-05-(-0.09)$. There was no significant effect of $\operatorname{sex}(Q=0.24 \mathrm{df}=1, p=$ 0.62 ) and all MS studies were conducted in rats (Table S2), preventing us from assessing the effect of species on performance in the probe trial. Moderator analyses found no significant effect of separation index $(\beta=-0.0032 \pm$ 0.0063, 95\% CI $=-0.016-(-0.0092), Z=-0.51, p=0.61$, $k=14)$. However, as with the latency to find the platform there was a significant effect of separation temp on outcomes in the probe trial with worse performance in rodents exposed to higher temp during the MS procedure $(\beta=$ $-0.33 \pm 0.12,95 \% \mathrm{CI}=-0.56-(-0.10), \quad Z=-2.80, \quad p=$ $0.05, k=8)$.

Similar to MS, rodents exposed to LBN showed significantly reduced time swimming in the correct quadrant (Fig. 2 bottom). This outcome had a large effect size that was highly significant (random effect: Hedge's $g=-0.82 \pm$ $0.35,95 \% \mathrm{CI}=-2.40-(-0.51), z=-2.4, p=0.02, k=7$; fixed effect: Hedge's $g=-0.61 \pm 0.17,95 \% \mathrm{CI}=-0.94$ $(-0.28), z=-3.6, p<0.0005, k=7)$. The heterogeneity within the LBN studies was large and statistically significant $\left(I^{2}=75 \%, Q=10, \mathrm{df}=6, p=0.001\right)$. There was evidence of significant publication bias for LBN studies (Supplemental information Fig. S4B, and egger's test: $p=0.02$ ). Using the trim-and-fill method to adjust for publication bias, findings remained significant in a fixed-effect (Hedge's $g=$ $-0.52,95 \% \mathrm{CI}=-0.85-(-0.20)$, but not a random-effect model (Hedge's $g=-0.65,95 \% \mathrm{CI}=-1.33-0.02)$. Metaregression demonstrated no significant effect of $\operatorname{sex}(Q=$ $2.3, \mathrm{df}=1, p=0.13)$ or species $(Q=1.3, \mathrm{df}=1, p=0.25)$.

\section{Novel object recognition (NOR)}

A forest plot summarizing the effects of handling (3 studies, $n=67$ rodents), MS (8 studies, $n=197$ rodents), and LBN (11 studies, $n=232$ rodents) on performance in the NOR test is shown in Fig. 3. Tests for subgroup differences found no significant differences in effect size using random effect $\left(\chi^{2}=2.45, \mathrm{df}=2, p=\right.$ $0.29)$, but significant differences using fixed-effect analysis $\left(\chi^{2}=7.94, \mathrm{df}=2, p=0.019\right)$. However, the relationship between the three paradigms was similar to outcomes in the latency to find platform and probe trial (Fig. 3).

Handling had a small effect size that was not significant (random effect: Hedges $g=0.10 \pm 0.40,95 \% \mathrm{CI}=-0.68-$ ( +0.88$), z=0.25, p=0.80, k=3$; fixed effect: Hedges $g=$ $0.17 \pm 0.24,95 \% \mathrm{CI}=-0.30-(+0.65), z=0.72, p=0.47$, $k=3$, Fig. 3, top). There was substantial heterogeneity between studies that did not reach statistical significance $\left(I^{2}=62 \%, Q=5, \mathrm{df}=2, p=0.071\right)$, most likely due to the small number of available studies (Table S2). The small number of studies also prevented us from assessing 


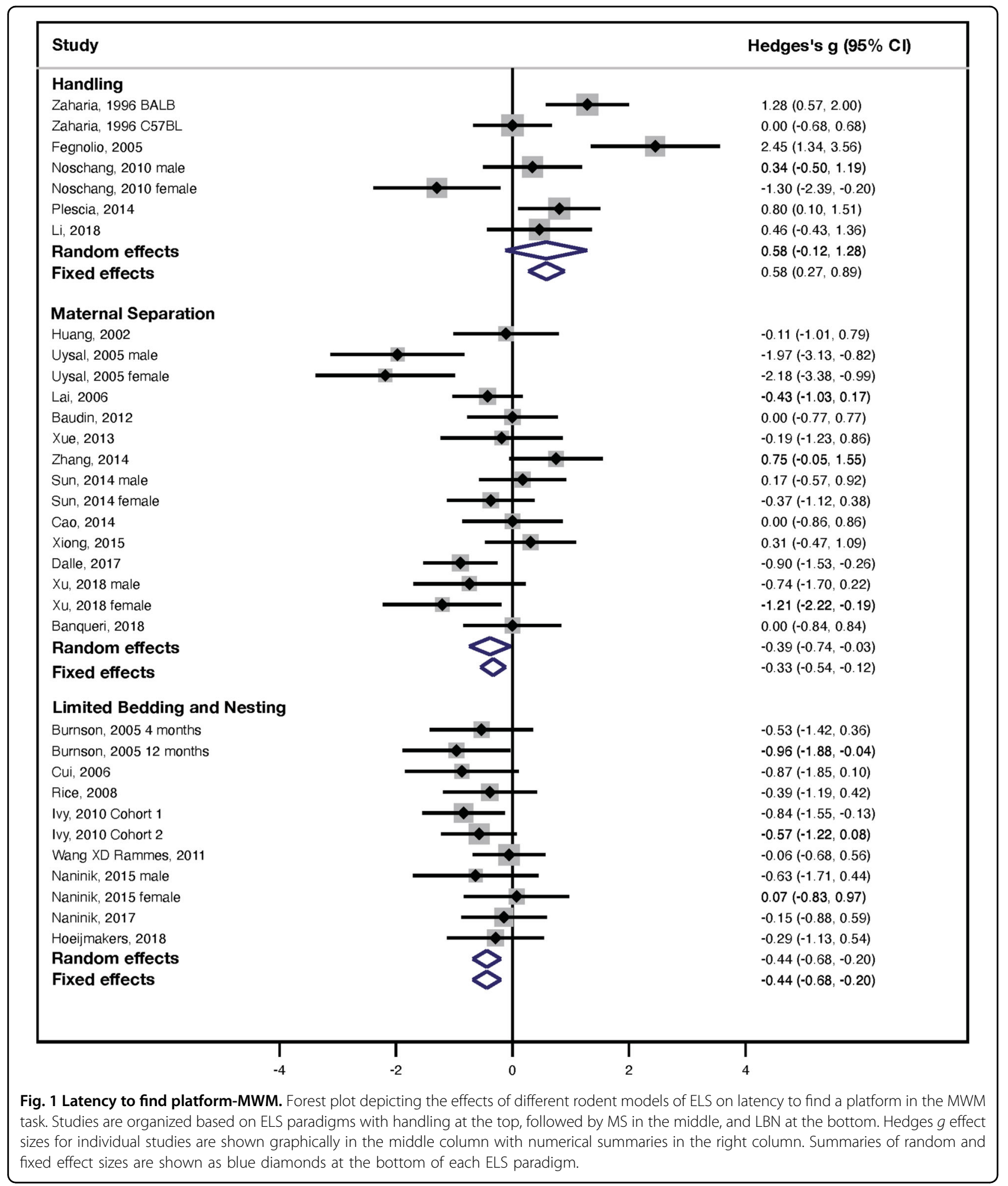

publication bias or the effects of sex and species on performance in the NOR test.

Rodents exposed to MS showed impaired memory in the NOR test that was moderate in size and statistically significant (random effect: Hedges $g=-0.59 \pm 0.26,95 \%$ $\mathrm{CI}=-1.1-(-0.08), \quad z=-2.27, \quad p=0.023, \quad k=8$; fixed effect: Hedges $g=-0.58 \pm 0.14,95 \% \mathrm{CI}=-0.86-(-0.29)$, $\mathrm{z}=-3.97, p<0.0005, k=8$, Fig. 3, middle). There was 


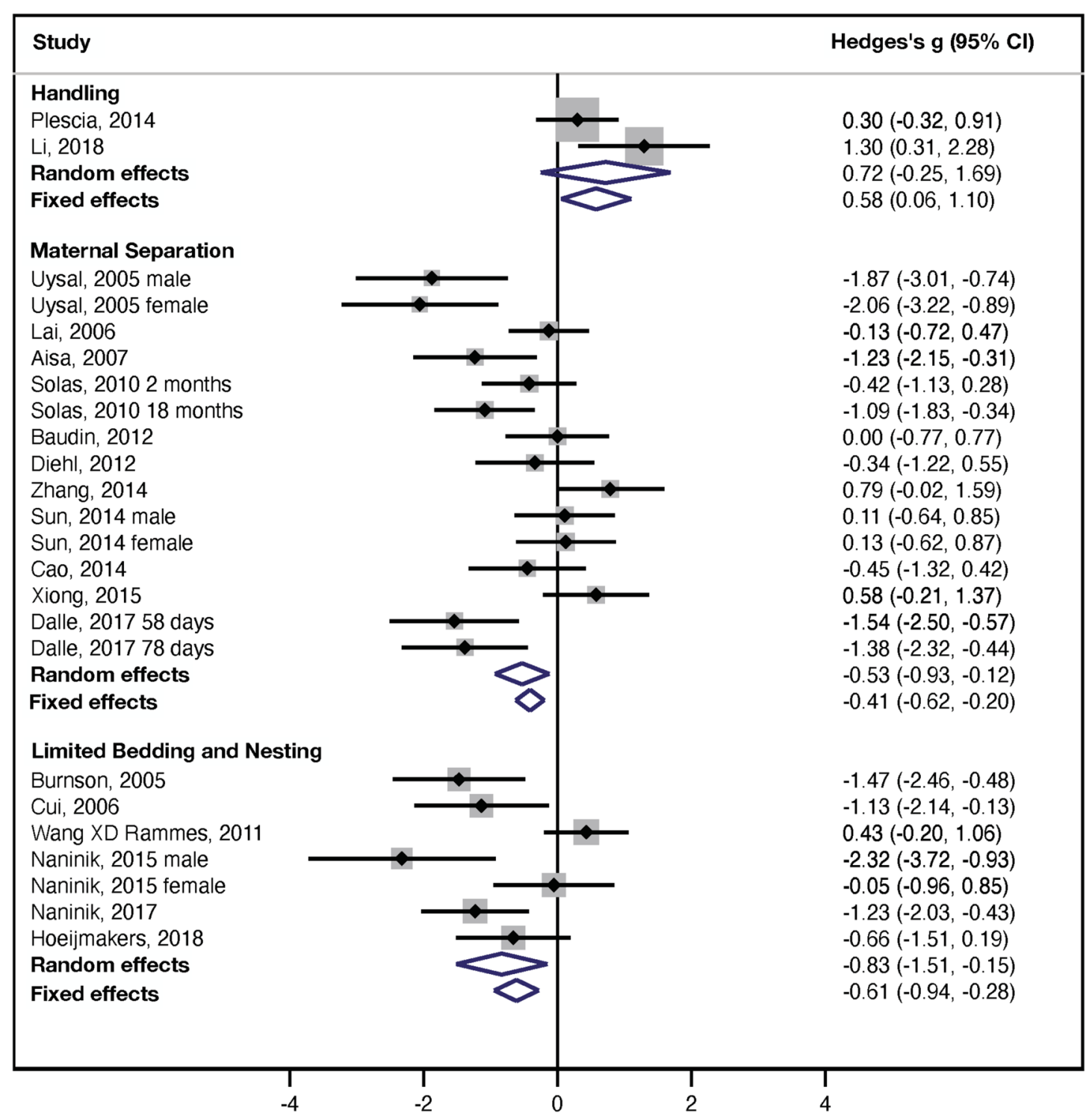

Fig. 2 Probe trial, MWM. Forest plot for the effects of handling, MS, and LBN on performance in the probe trial of the MWM. Studies are organized based on ELS paradigms with the effect size for individual studies shown graphically in the middle column and numerical summaries available in the right column. Overall random and fixed effect sizes are shown as blue diamonds at the bottom of each ELS paradigm.

substantial heterogeneity between studies $\left(I^{2}=69 \%, Q=\right.$ $22, \mathrm{df}=7, p=0.0022)$ but no evidence of publication bias based on funnel plot asymmetry (Supplemental information Fig. S5A) or the Egger's test $(p=0.69)$. There was no significant effect of species $(Q=0.15, \mathrm{df}=1, p=0.70)$ or sex: $(\mathrm{Q}=0.95, \mathrm{df}=1, \quad p=0.33)$. Moderator analyses found a significant effect of separation index on performance in the NOR, with longer separation associated with worse outcomes $(\beta=-0.047 \pm 0.017,95 \% \mathrm{CI}=-0.080$ $(-0.013), Z=-2.73, p=0.0064, k=8)$. No analysis was done for separation temperature because only 4 studies reported it (Table S1).
Exposure to LBN caused a significant impairment in the NOR test (random effect: Hedges $g=-0.54 \pm 0.14,95 \%$ $\mathrm{CI}=-0.82-(-0.26), z=-3.79, p<0.0005, k=11$; fixed effect: Hedges $g=-0.54 \pm 0.13,95 \% \mathrm{CI}=-0.80-(-0.28)$, $z=-4.06, p<0.0005, k=11$, Fig. 3, bottom). Heterogeneity within the LBN studies was small and non-significant $\left(I^{2}=\right.$ $11 \%, Q=11, \mathrm{df}=10, p=0.34)$. There was no evidence of publication bias based on funnel plot asymmetry (Supplemental information Fig. S5B) or the Egger's test $(p=0.96)$ and no significant effect species $(Q=1.05, \mathrm{df}=1, p=0.31)$. Only one study was conducted in females, preventing us from assessing the effect of sex (Table S2). 


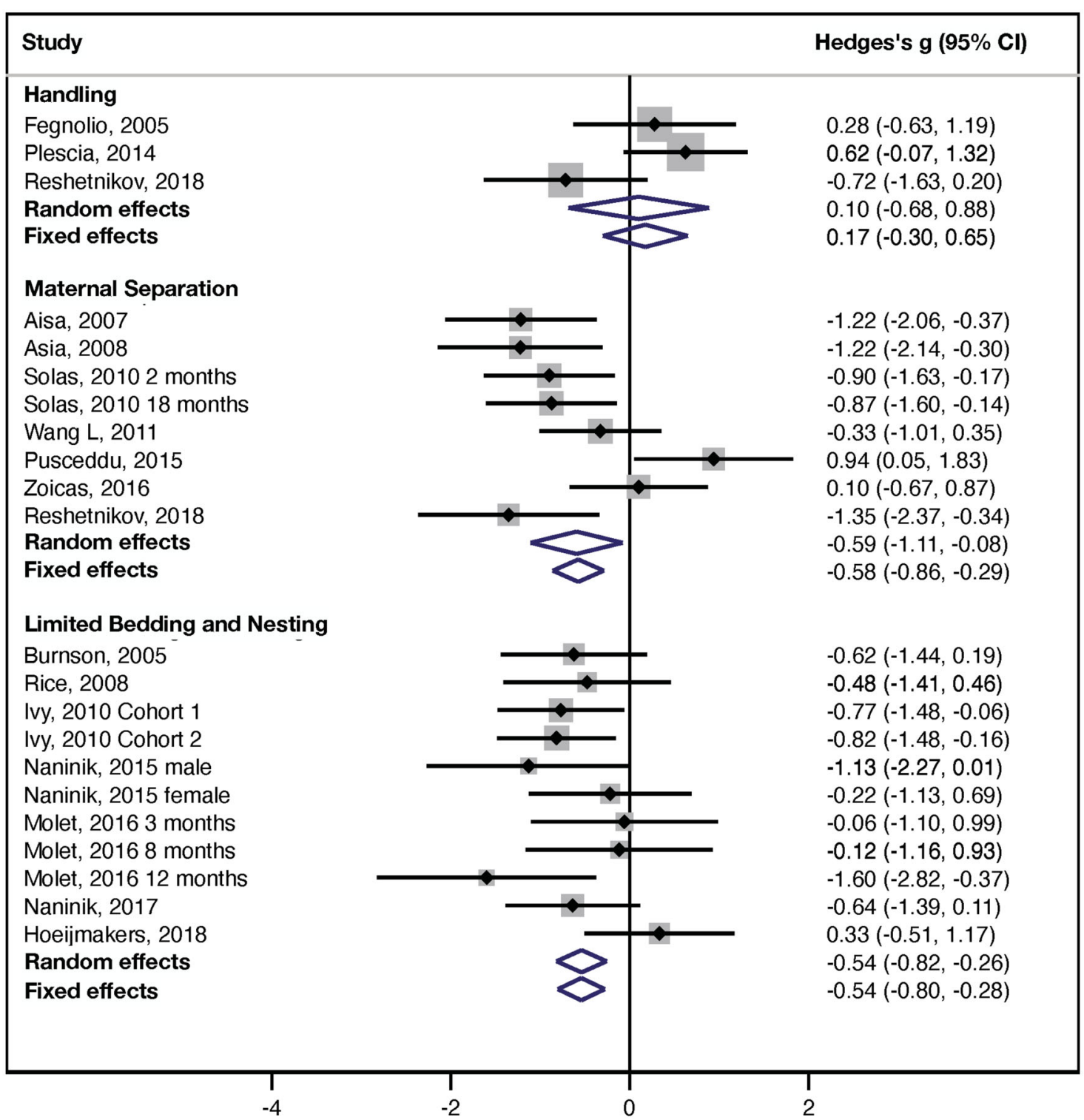

Fig. 3 Forest plot-NOR. Forest plot examining the effects of handling, MS, and LBN on NOR test. Studies are organized based on ELS paradigms. Hedges $g$ effect sizes for individual studies are shown graphically in the middle column with numerical summaries provided in the right column. Summaries of random and fixed effect sizes are shown as blue diamonds at the bottom for each ELS paradigm.

\section{Contextual fear conditioning (CFC)}

A forest plot summary for the overall effect sizes of handling (4 studies, $n=92$ rodents), MS (12 studies, $n=$ 299 rodents), and LBN (3 studies, $n=60$ rodents) on freezing behavior in the CFC are shown in Fig. 4. Unlike outcomes in the MWM and NOR, tests for subgroup differences did not reveal significant differences in effect size (Hedge's $g$ ) between the three experimental paradigms (random effect: $\chi^{2}=1.52, \mathrm{df}=2, p=0.4$; fixed effect: $\chi^{2}=0.34, \mathrm{df}=2, p=0.84$, Fig. 4).

In contrast to the improved cognition in the MWM and the lack of impact in the NOR, exposure to handling reduced contextual freezing suggesting impaired hippocampal-dependent memory. The effect size was moderate and did not reach statistical significance using random-effect analysis, most likely due to the small number of available studies (Hedge's $g=$ $-0.48 \pm 0.33,95 \% \mathrm{CI}=-1.12-(+0.17), z=-1.44, p=$ $0.15, k=4)$. The effect of handling on CFC was significant when the fixed-effect analysis was performed (Hedge's $g=-0.41 \pm 0.21,95 \% \mathrm{CI}=-0.83-(-0.001)$, $z=-1.97, p=0.049, k=4$, Fig. 4 top). There was a large heterogeneity that did not reach statistical significance $\left(I^{2}=57 \%, Q=6.92, \mathrm{df}=3, p=0.075\right)$ and too 


\begin{tabular}{|c|c|}
\hline Study & Hedges's g (95\% Cl) \\
\hline \multicolumn{2}{|l|}{ Handling } \\
\hline Kosten, 2006 male & $-0.87(-1.91,0.18)$ \\
\hline Kosten, 2006 female & $-0.09(-1.08,0.90)$ \\
\hline Guijarro, 2007 & $0.10(-0.54,0.75)$ \\
\hline Couto-Pereira, 2019 & $-1.17(-1.98,-0.36)$ \\
\hline Random effects & $-0.48(-1.12,0.17)$ \\
\hline Fixed effects & $-0.41(-0.83,-0.00)$ \\
\hline \multicolumn{2}{|l|}{ Maternal Separation } \\
\hline Kosten, 2006 male & $0.15(-0.84,1.15)$ \\
\hline Kosten, 2006 female & $-0.92(-1.97,0.12)$ \\
\hline Guijarro, 2007 & $-0.07(-0.76,0.62)$ \\
\hline Sun, 2014 male & $-0.34(-1.33,0.65)$ \\
\hline Sun, 2014 female & $-1.00(-1.99,-0.00)$ \\
\hline Chocyk, 2014 male & $-0.75(-1.38,-0.12)$ \\
\hline Chocyk, 2014 female & $-0.93(-1.57,-0.29)$ \\
\hline Diehl, 2014 male & $2.73(1.41,4.05)$ \\
\hline Diehl, 2014 female & $9.63(6.17,13.09)$ \\
\hline Xiong, 2014 & $-0.25(-1.18,0.68)$ \\
\hline Xiong, 2015 & $0.27(-0.43,0.97)$ \\
\hline Couto-Pereira, 2019 & $-0.53(-1.29,0.23)$ \\
\hline Random effects & $0.05(-0.58,0.67)$ \\
\hline Fixed effects & $-0.28(-0.53,-0.03)$ \\
\hline \multicolumn{2}{|l|}{ Limited Bedding and Nesting } \\
\hline Kanatsou, 2017 & $0.09(-0.75,0.93)$ \\
\hline Manzano-Nieves, 2018 male & $-0.42(-1.30,0.46)$ \\
\hline Manzano-Nieves, 2018 female & $-0.86(-1.77,0.05)$ \\
\hline Random effects & $-0.37(-0.91,0.16)$ \\
\hline Fixed effects & $-0.37(-0.88,0.13)$ \\
\hline
\end{tabular}

Fig. 4 Forest plot-CFC. Forest plot for the effects of handling, MS and LBN on freezing behavior in the CFC test. Studies are organized based on ELS paradigms with the effect size for individual studies shown graphically in the middle column and numerical summaries available in the right column. Summaries of random and fixed effect sizes are shown as blue diamonds at the bottom of each ELS paradigm.

few studies were available to assess publication bias or the effects of sex and species.

MS also reduced contextual freezing, an effect that was not significant for random effect (Hedges $g=0.05 \pm 0.32$, $95 \% \mathrm{CI}=-0.58-(+0.67), \mathrm{z}=0.15, p=0.88, k=12)$, but significant for fixed-effect analysis (Hedges $g=-0.28 \pm$ $0.13,95 \% \mathrm{CI}=-0.53-(-0.03), \mathrm{z}=-2.24, p=0.025, k=$ 12, Fig. 4, middle). As with other studies involving MS, there was large and highly significant heterogeneity between studies $\left(I^{2}=83 \%, Q=64.8, \mathrm{df}=11, p<0.0005\right)$. There was also substantial evidence for publication bias based on funnel plot asymmetry and the Egger's test $(p=0.006)$ that were highly influenced by 1 outlier study (46) (Supplemental information Fig. S6). We were unable to assess the effect of species because all studies were conducted in rats (Table S2) and there was no significant effect of sex $(Q=$ $0.08, \mathrm{df}=1, p=0.78$ ). Moderator analyses of MS studies found no significant effect of separation index $(\beta=$ $-0.015 \pm 0.0098,95 \% \mathrm{CI}=-0.035-(0.041), Z=-1.55, p=$ $0.12, k=12)$ or separation temp $(\beta=0.063 \pm 0.087,95 \% \mathrm{CI}$ $=-0.11-(0.23), \mathrm{Z}=0.72, p=0.47, k=10)$.

Rodents exposed to LBN also showed reduced contextual freezing, with a moderate effect size that did not reach significance most likely due to the small number of 
available studies (random effect: Hedge's $g=-0.37 \pm 0.27$, $95 \% \mathrm{CI}=-0.91-(+0.16), \mathrm{z}=-1.36, p=0.17, \quad k=3$; fixed effect: Hedge's $g=-0.37 \pm 0.26,95 \% \mathrm{CI}=-0.88-$ ( +0.13$), z=-1.44, p=0.15, k=3$, Fig. 4 bottom). Heterogeneity between studies was small and did not reach statistical significance $\left(I^{2}=12 \%, Q=2.27, \mathrm{df}=2, p=\right.$ 0.32 ) and too few studies were available to assess publication bias or the effects of sex or species (Table S2).

\section{Discussion}

This is the first meta-analysis that examines the effects of different rodent models of ELS on spatial learning providing several new insights to some key questions in the field. For example, we show that MS and LBN cause similar cognitive deficits in the MWM latency to find the platform, the probe trial, and the NOR test (Summarized in Fig. S2). The effect sizes of MS and LBN in these tasks were mostly moderate (Hedge's $g=-0.3-(-0.6)$, except for large effect size in the probe trial for LBN (Hedge's $g=-0.82$ ). In contrast, exposure to handing showed improved performance in the latency to find platform and probe trial of the MWM, with no clear difference compared to control condition in the NOR (Fig. S2). These results demonstrate both quantitative and qualitative differences between handling and MS and LBN in the MWM and NOR and are consistent with previous work showing that handling causes different outcomes in stress reactivity ${ }^{33,37}$ and sensitivity to pain compared to $\mathrm{MS}^{30}$. Sustained elevation of $\mathrm{CRH}$ in the hippocampus has been shown to play a central role in inducing synaptic abnormalities and spatial-learning deficits in rodents exposed to $\mathrm{LBN}^{12,38}$, but its role in modifying hippocampal function in MS and handling has not been studied extensively. Nevertheless, Wang et al. found increased CRH levels in the hippocampus in rats exposed to MS, and administrating the CRH receptor antagonist (CP-154526) improved performance in the MWM and NOR in rats exposed to $\mathrm{MS}^{39}$. To the best of our knowledge, no group has yet shown that handling causes a reduction in CRH levels in the hippocampus, but work by Fenoglio et al. found that transient administration of CRH receptor 1 antagonist to control pups from P10-17, enhanced performance in the MWM and NOR to levels seen in handled animals. A similar procedure did not affect cognitive performance in handled animals, consistent with the notion that $\mathrm{CRH}$ levels are low in this group ${ }^{40}$. Additional studies are therefore warranted to compare CRH levels in the dorsal and ventral hippocampus (see below), across these three models of ELS.

One of the most intriguing findings of our analysis is that exposure to handling causes similar deficits in contextual freezing compared to LBN and MS (Fig. S2). This result needs to be interpreted with caution given the relatively small number of studies contributing to this outcome and that the impacts of handling and MS were only significant using fixed-effect analysis. Nevertheless, it demonstrates that handling shares some similarities with other ELS paradigms and raises the intriguing possibility that all three ELS paradigms cause similar deficits in the ventral hippocampus. This assertion is consistent with data showing that the ventral hippocampus plays an important role in contextual freezing ${ }^{8,22-25}$ and provides a parsimonious explanation for these seemingly conflicting results. Additional support comes from studies showing that the offspring of low licking and grooming (LG) dams show reduced longterm potentiation (LTP) and poor spatial learning in the dorsal hippocampus, but increased LTP in the ventral hippocampus ${ }^{41}$ and enhanced contextual freezing compared to offspring of high-LG ${ }^{42}$. Important differences in the effects of prenatal stress on the dorsal versus the ventral hippocampus have also been reported ${ }^{43}$. However, given the role that other brain regions, such as the prefrontal cortex, play in these tasks ${ }^{17,18,26-28}$, it is also possible that changes in these other areas and not the hippocampus are responsible for these behavioral outcomes.

The heterogeneity in LBN studies was significantly lower compared to both MS and handling studies across 3 of the 4 cognitive tests. Large heterogeneity was also found in a recent meta-analysis examining the effects of MS on anxiety-like behavior ${ }^{31}$. This is not surprising given the lack of standardization in the separation procedure associated with the MS and handling paradigms (e.g., number of days, length of separation, temp, single vs. whole litter separation). In addition, regular human contact in the MS and handling procedures may also increase variability due to the effects of the sex of the researcher and his/her experience scruffing and transferring animals ${ }^{31}$. Indeed, moderator analyses focusing on MS studies found that longer separation procedures (i.e., greater separation index) were associated with more severe cognitive outcomes in the NOR, but not MWM. Surprisingly, we found that incubating pups at higher temp during the separation procedure was associated with worse cognitive outcomes in the MWM (both latencies to find platform and probe trial) and were not able to examine this in the NOR because of the small number of studies available. This was an unexpected outcome because of the known sensitivity of rodent pups to hypothermia ${ }^{44}$ and will require additional replication.

A previous meta-analysis has found that maternal separation increases anxiety-like behavior in rats, but not mice $^{31}$. This was not the case for hippocampal-dependent tasks where most of the outcomes were similar in rats and mice. The only exception was a performance in the latency to find a platform where rats exposed to LBN were significantly more affected than mice exposed to LBN (Fig. 1). A systematic review without meta-analysis conducted by Loi et al. reported that males had more cognitive deficits in non-stressful learning paradigms compared to female rodents $^{13}$. This was confirmed for NOR in rodents exposed to MS, but overall there was no clear sex effect on 
hippocampal-dependent function. These different outcomes might be due to the inclusion of non-hippocampal learning tests in the Loi et al. study and the more quantitative approach used in this study.

\section{Conclusions}

This work provides several new insights on the impact of different rodent models of ELS on spatial learning. First, LBN and MS, cause similar deficits in tasks such as the MWM and NOR that rely heavily on the dorsal hippocampus. In contrast, handling improved performance in the MWM and had no significant effect in the NOR. Second, all ELS paradigms, reduced contextual freezing, suggesting similar abnormalities in the ventral hippocampus and/or other brain regions. Third, heterogeneity was significantly lower in LBN compared to handling and MS. Fourth, ELS causes similar cognitive deficits in male and female rodents with no differences in the sensitivity between mice and rats.

\section{Acknowledgements}

This work was supported by: NIMH 1R56MH114833, NIMH R01MH1 1833, NIMH R01MH119164.

\section{Conflict of interest}

Dr. M.H.B. receives research support from Biohaven Pharmaceuticals, Janssen Pharmaceuticals, Emalex Pharmaceuticals, and Neurocrine Biosciences. All other authors (M.R., D.W., V.A.Q., and A.K.) reported no biomedical financial interests or potential conflicts of interest.

\section{Publisher's note}

Springer Nature remains neutral with regard to jurisdictional claims in published maps and institutional affiliations.

Supplementary information The online version contains supplementary material available at https://doi.org/10.1038/s41398-021-01352-4.

Received: 26 September 2020 Revised: 20 March 2021 Accepted: 1 April 2021

Published online: 20 April 2021

\section{References}

1. Teicher, M. H. \& Samson, J. A. Annual research review: enduring neurobiological effects of childhood abuse and neglect. J. Child Psychol. Psychiatry 57, 241-266 (2016).

2. Frodl, T. et al. Childhood adversity impacts on brain subcortical structures relevant to depression. J. Psychiatr. Res. 86, 58-65 (2017).

3. Lambert, H. K. et al. Altered development of hippocampus-dependent associative learning following early-life adversity. Dev. Cogn. Neurosci. 38, 100666 (2019).

4. De Bellis, M. D., Woolley, D. P. \& Hooper, S. R. Neuropsychological findings in pediatric maltreatment: relationship of PTSD, dissociative symptoms, and abuse/neglect indices to neurocognitive outcomes. Child Maltreat. 18, 171-183 (2013).

5. Khalaf-Nazzal, R. \& Francis, F. Hippocampal development-old and new findings. Neuroscience 248, 225-242 (2013).

6. Wei, L. et al. Early-life stress perturbs key cellular programs in the developing mouse hippocampus. Dev. Neurosci. 37, 476-488 (2015).

7. Brunson, K. L., Chen, Y., Avishai-Eliner, S. \& Baram, T. Z. Stress and the developing hippocampus: a double-edged sword? Mol. Neurobiol. 27, 121-136 (2003).

8. Fanselow, M. S. \& Dong, H. W. Are the dorsal and ventral hippocampus functionally distinct structures? Neuron 65, 7-19 (2010).
9. Leuner, B. \& Gould, E. Structural plasticity and hippocampal function. Annu. Rev. Psychol. 61, 111-140 (2010).

10. White, J. D. \& Kaffman, A. The moderating effects of sex on consequences of childhood maltreatment: from clinical studies to animal models. Front. Neurosci. 13, 1082 (2019).

11. Li, G. \& Pleasure, S. J. Morphogenesis of the dentate gyrus: what we are learning from mouse mutants. Dev. Neurosci. 27, $93-99$ (2005).

12. Chen, Y., Andres, A. L., Frotscher, M. \& Baram, T. Z. Tuning synaptic transmission in the hippocampus by stress: the CRH system. Front. Cell. Neurosci. 6, 13 (2012).

13. Loi, M. et al. Effects of early-life stress on cognitive function and hippocampal structure in female rodents. Neuroscience 342, 101-119 (2015).

14. Tractenberg, S. G. et al. An overview of maternal separation effects on behavioural outcomes in mice: evidence from a four-stage methodological systematic review. Neurosci. Biobehav. Rev. 68, 489-503 (2016).

15. Barrera-Ocampo, A. \& Lopera, F. Amyloid-beta immunotherapy: the hope for Alzheimer disease? Colomb. Med. 47, 203-212 (2016).

16. Murthy, S. \& Gould, E. Early life stress in rodents: animal models of illness or resilience? Front. Behav. Neurosci. 12, 157 (2018).

17. Zemla, R. \& Basu, J. Hippocampal function in rodents. Curr. Opin. Neurobiol. 43 187-197 (2017).

18. DeVito, L. M. \& Eichenbaum, H. Distinct contributions of the hippocampus and medial prefrontal cortex to the "what-where-when" components of episodiclike memory in mice. Behav. Brain Res. 215, 318-325 (2010).

19. Moser, M. B. \& Moser, E. I. Functional differentiation in the hippocampus. Hippocampus 8, 608-619 (1998).

20. Moser, M. B., Moser, E. l., Forrest, E., Andersen, P. \& Morris, R. G. Spatial learning with a minislab in the dorsal hippocampus. Proc. Natl Acad. Sci. USA 92 9697-9701 (1995)

21. Jung, M. W., Wiener, S. I. \& McNaughton, B. L. Comparison of spatial firing characteristics of units in dorsal and ventral hippocampus of the rat. J. Neurosci. 14, 7347-7356 (1994).

22. Huff, M. L., Emmons, E. B., Narayanan, N. S. \& LaLumiere, R. T. Basolateral amygdala projections to ventral hippocampus modulate the consolidation of footshock, but not contextual, learning in rats. Learn Mem. 23, 51-60 (2016).

23. Jimenez, J. C. et al. Anxiety cells in a hippocampal-hypothalamic circuit. Neuron 97, 670-683 e676 (2018)

24. Zhu, H. et al. Chemogenetic inactivation of ventral hippocampal glutamatergic neurons disrupts consolidation of contextual fear memory. Neuropsychopharmacology 39, 1880-1892 (2014).

25. Rudy, J. W. \& Matus-Amat, P. The ventral hippocampus supports a memory representation of context and contextual fear conditioning: implications for a unitary function of the hippocampus. Behav. Neurosci. 119, 154-163 (2005).

26. Steadman, P. E. et al. Disruption of oligodendrogenesis impairs memory consolidation in adult mice. Neuron 105, 150-164 e156 (2020).

27. Giustino, T. F. \& Maren, S. The role of the medial prefrontal cortex in the conditioning and extinction of fear. Front. Behav. Neurosci. 9, 298 (2015).

28. Warburton, E. C. \& Brown, M. W. Neural circuitry for rat recognition memory. Behav. Brain Res. 285, 131-139 (2015).

29. Kaffman, A., White, J. D., Wei, L., Johnson, F. K. \& Krystal, J. H. Enhancing the utility of preclinical research in neuropsychiatry drug development. Methods Mol. Biol. 2011, 3-22 (2019).

30. Chen, L. \& Jackson, T. Early maternal separation and responsiveness to thermal nociception in rodent offspring: A meta-analytic review. Behav. Brain Res. 299, 42-50 (2016).

31. Wang, D., Levine, J. L. S., Avila-Quintero, V., Bloch, M. \& Kaffman, A. Systematic review and meta-analysis: effects of maternal separation on anxiety-like behavior in rodents. Transl. Psychiatry 10, 174 (2020).

32. Walker, C. D. et al. Chronic early life stress induced by limited bedding and nesting (LBN) material in rodents: critical considerations of methodology, outcomes and translational potential. Stress 20, 421-448 (2017).

33. Pryce, C. R. \& Feldon, J. Long-term neurobehavioural impact of the postnatal environment in rats: manipulations, effects and mediating mechanisms. Neurosci. Biobehav. Rev. 27, 57-71 (2003).

34. Vesterinen, H. M. et al. Meta-analysis of data from animal studies: a practical guide. J. Neurosci. Methods 221, 92-102 (2014).

35. von Hippel, P. T. The heterogeneity statistic I(2) can be biased in small metaanalyses. BMC Med. Res. Methodol. 15, 35 (2015).

36. Israel, H. \& Richter, R. R. A guide to understanding meta-analysis. J. Orthop. Sports Phys. Ther. 41, 496-504 (2011). 
37. Meaney, M. J. Maternal care, gene expression, and the transmission of individual differences in stress reactivity across generations. Annu. Rev. Neurosci. 24, 1161-1192 (2001).

38. Chen, Y. \& Baram, T. Z. Toward understanding how early-life stress reprograms cognitive and emotional brain networks. Neuropsychopharmacology 41, 197-206 (2016).

39. Wang, A. et al. Epigenetic upregulation of corticotrophin-releasing hormone mediates postnatal maternal separation-induced memory deficiency. PLoS ONE 9, e94394 (2014).

40. Fenoglio, K. A. et al. Enduring, handling-evoked enhancement of hippocampal memory function and glucocorticoid receptor expression involves activation of the corticotropin-releasing factor type 1 receptor. Endocrinology 146, 4090-4096 (2005)

41. Nguyen, H. B., Bagot, R. C., Diorio, J., Wong, T. P. \& Meaney, M. J. Maternal care differentially affects neuronal excitability and synaptic plasticity in the dorsal and ventral hippocampus. Neuropsychopharmacology 40, 1590-1599 (2015).

42. Champagne, D. L. et al. Maternal care and hippocampal plasticity: evidence for experience-dependent structural plasticity, altered synaptic functioning, and differential responsiveness to glucocorticoids and stress. J. Neurosci. 28 6037-6045 (2008).

43. Grigoryan, G. \& Segal, M. Lasting differential effects on plasticity induced by prenatal stress in dorsal and ventral hippocampus. Neural Plast. 2016, 2540462 (2016).

44. Lagerspetz, K. The postnatal development of homoiothermy and cold resistance in mice. Experientia 18, 282-284 (1962).

45. Aisa, B., Tordera, R., Lasheras, B., Del Rio, J. \& Ramirez, M. J. Cognitive impairment associated to HPA axis hyperactivity after maternal separation in rats. Psychoneuroendocrinology 32, 256-266 (2007).

46. Aisa, B., Tordera, R., Lasheras, B., Del Rio, J. \& Ramirez, M. J. Effects of maternal separation on hypothalamic-pituitary-adrenal responses, cognition and vulnerability to stress in adult female rats. Neuroscience 154, 1218-1226 (2008).

47. Banqueri, M., Mendez, M. \& Arias, J. L. Why are maternally separated females inflexible? Brain activity pattern of COx and c-Fos. Neurobiol. Learn. Mem. 155, 30-41 (2018).

48. Baudin, A. et al. Maternal deprivation induces deficits in temporal memory and cognitive flexibility and exaggerates synaptic plasticity in the rat medial prefrontal cortex. Neurobiol. Learn. Mem. 98, 207-214 (2012).

49. Brunson, K. L. et al. Mechanisms of late-onset cognitive decline after early-life stress. J. Neurosci. 25, 9328-9338 (2005).

50. Cao, X. et al. The timing of maternal separation affects Morris water maze performance and long-term potentiation in male rats. Dev. Psychobiol. 56, 1102-1109 (2014).

51. Chocyk, A. et al. The effects of early-life adversity on fear memories in adolescent rats and their persistence into adulthood. Behav. Brain Res. 264, 161-172 (2014).

52. Couto-Pereira, N. S. et al. Resilience and vulnerability to trauma: early life interventions modulate aversive memory reconsolidation in the dorsal hippocampus. Front. Mol. Neurosci. 12, 134 (2019).

53. Cui, M. et al. Enriched environment experience overcomes the memory deficits and depressive-like behavior induced by early life stress. Neurosci. Lett. 404, 208-212 (2006).

54. Dalle, E., Daniels, W. M. U. \& Mabandla, M. V. Fluvoxamine maleate effects on dopamine signaling in the prefrontal cortex of stressed Parkinsonian rats: Implications for learning and memory. Brain Res. Bull. 132, 75-81 (2017).

55. Diehl, L. A. et al. Long-lasting effects of maternal separation on an animal model of post-traumatic stress disorder: effects on memory and hippocampal oxidative stress. Neurochem. Res. 37, 700-707 (2012).

56. Diehl, L. A. et al. Contextual fear conditioning in maternal separated rats: the amygdala as a site for alterations. Neurochem. Res. 39, 384-393 (2014).

57. Guijarro, J. Z. et al. Effects of brief and long maternal separations on the HPA axis activity and the performance of rats on context and tone fear conditioning. Behav. Brain Res. 184, 101-108 (2007).

58. Hoeijmakers, L. et al. Early-life stress does not aggravate spatial memory or the process of hippocampal neurogenesis in adult and middle-aged APP/PS1 mice. Front. Aging Neurosci. 10, 61 (2018).

59. Huang, L. T. et al. Maternal deprivation stress exacerbates cognitive deficits in immature rats with recurrent seizures. Epilepsia 43, 1141-1148 (2002).

60. Ivy, A. S. et al. Hippocampal dysfunction and cognitive impairments provoked by chronic early-life stress involve excessive activation of $\mathrm{CRH}$ receptors. J. Neurosci. 30, 13005-13015 (2010).
61. Kanatsou, S. et al. Overexpression of mineralocorticoid receptors in the mouse forebrain partly alleviates the effects of chronic early life stress on spatial memory, neurogenesis and synaptic function in the dentate gyrus. Front. Cell. Neurosci. 11, 132 (2017).

62. Kosten, T. A., Lee, H. J. \& Kim, J. J. Early life stress impairs fear conditioning in adult male and female rats. Brain Res. 1087, 142-150 (2006).

63. Lai, M. C. et al. Effect of neonatal isolation on outcome following neonatal seizures in rats-the role of corticosterone. Epilepsy Res. 68, 123-136 (2006).

64. Li, H., Ishikawa, C. \& Shiga, T. Effects of postnatal handling on adult behavior and brain mRNA expression of serotonin receptor, brain-derived neurotrophic factor and GABA-A receptor subunit. Int. J. Dev. Neurosci. 68, 17-25 (2018).

65. Manzano-Nieves, G., Gaillard, M., Gallo, M. \& Bath, K. G. Early life stress impairs contextual threat expression in female, but not male, mice. Behav. Neurosci. 132, 247-257 (2018).

66. Molet, J. et al. MRI uncovers disrupted hippocampal microstructure that underlies memory impairments after early-life adversity. Hippocampus 26, 1618-1632 (2016).

67. Naninck, E. F. et al. Chronic early life stress alters developmental and adult neurogenesis and impairs cognitive function in mice. Hippocampus $\mathbf{2 5}$ 309-328 (2015).

68. Naninck, E. F. et al. Early micronutrient supplementation protects against early stress-induced cognitive impairments. FASEB J. 31, 505-518 (2017).

69. Noschang, C. G. et al. Neonatal handling impairs spatial memory and leads to altered nitric oxide production and DNA breaks in a sex specific manner. Neurochem. Res. 35, 1083-1091 (2010).

70. Plescia, F. et al. Early handling effect on female rat spatial and non-spatial learning and memory. Behav. Process. 103, 9-16 (2014).

71. Pusceddu, M. M. et al. n-3 PUFAs have beneficial effects on anxiety and cognition in female rats: Effects of early life stress. Psychoneuroendocrinology 58, 79-90 (2015).

72. Reshetnikov, $\mathrm{V}$. V. et al. The long-term effects of early postnatal stress on cognitive abilities and expression of genes of the glutamatergic system in mice. Neurochem. J. 12, 142-151 (2018).

73. Rice, C. J., Sandman, C. A., Lenjavi, M. R. \& Baram, T. Z. A novel mouse model for acute and long-lasting consequences of early life stress. Endocrinology 149, 4892-4900 (2008).

74. Solas, M. et al. Interactions between age, stress and insulin on cognition: implications for Alzheimer's disease. Neuropsychopharmacology 35, 1664-1673 (2010).

75. Sun, X. M., Tu, W. Q., Shi, Y. W., Xue, L. \& Zhao, H. Female-dependent impaired fear memory of adult rats induced by maternal separation, and screening of possible related genes in the hippocampal CA1. Behav. Brain Res. 267, 111-118 (2014).

76. Uysal, N. et al. Effects of maternal deprivation on melatonin production and cognition in adolescent male and female rats. Neuro. Endocrinol. Lett. 26, 555-560 (2005).

77. Wang, L., Jiao, J. \& Dulawa, S. C. Infant maternal separation impairs adult cognitive performance in BALB/CJ mice. Psychopharmacology 216, 207-218 (2011).

78. Wang, X. D. et al. Forebrain CRF(1) modulates early-life stress-programmed cognitive deficits. J. Neurosci. 31, 13625-13634 (2011).

79. Xiong, G. J., Yang, Y., Wang, L. P., Xu, L. \& Mao, R. R. Maternal separation exaggerates spontaneous recovery of extinguished contextual fear in adult female rats. Behav. Brain Res. 269, 75-80 (2014).

80. Xiong, G. J., Yang, Y., Cao, J., Mao, R. R. \& Xu, L. Fluoxetine treatment reverses the intergenerational impact of maternal separation on fear and anxiety behaviors. Neuropharmacology 92, 1-7 (2015).

81. $\mathrm{Xu}, \mathrm{H}$. et al. Sex differences in associations between maternal deprivation and alterations in hippocampal calcium-binding proteins and cognitive functions in rats. Behav. Brain Funct. 14, 10 (2018).

82. Xue, X., Shao, S., Wang, W. \& Shao, F. Maternal separation induces alterations in reversal learning and brain-derived neurotrophic factor expression in adult rats. Neuropsychobiology 68, 243-249 (2013).

83. Zaharia, M. D., Kulczycki, J., Shanks, N., Meaney, M. J. \& Anisman, H. The effects of early postnatal stimulation on Morris water-maze acquisition in adult mice: genetic and maternal factors. Psychopharmacology 128, 227-239 (1996).

84. Zhang, $X$. et al. Early deprivation reduced anxiety and enhanced memory in adult male rats. Brain Res. Bull. 108, 44-50 (2014).

85. Zoicas, I. \& Neumann, I. D. Maternal separation facilitates extinction of social fear in adult male mice. Behav. Brain Res. 297, 323-328 (2016). 